\title{
Bimodal oscillations in nephron autoregulation
}

\section{Sosnovtseva, Olga; Pavlov, A.N.; Mosekilde, Erik; Holstein-Rathlou, N.H.}

\section{Published in:}

Physical Review E. Statistical, Nonlinear, and Soft Matter Physics

Link to article, DOI:

10.1103/PhysRevE.66.061909

Publication date:

2002

Document Version

Publisher's PDF, also known as Version of record

Link back to DTU Orbit

Citation (APA):

Sosnovtseva, O., Pavlov, A. N., Mosekilde, E., \& Holstein-Rathlou, N. H. (2002). Bimodal oscillations in nephron autoregulation. Physical Review E. Statistical, Nonlinear, and Soft Matter Physics, 66(6), 061909.

https://doi.org/10.1103/PhysRevE.66.061909

\section{General rights}

Copyright and moral rights for the publications made accessible in the public portal are retained by the authors and/or other copyright owners and it is a condition of accessing publications that users recognise and abide by the legal requirements associated with these rights.

- Users may download and print one copy of any publication from the public portal for the purpose of private study or research.

- You may not further distribute the material or use it for any profit-making activity or commercial gain

- You may freely distribute the URL identifying the publication in the public portal

If you believe that this document breaches copyright please contact us providing details, and we will remove access to the work immediately and investigate your claim. 


\title{
Bimodal oscillations in nephron autoregulation
}

\author{
O. V. Sosnovtseva, ${ }^{1,2}$ A. N. Pavlov, ${ }^{1}$ E. Mosekilde, ${ }^{2}$ and N.-H. Holstein-Rathlou ${ }^{3}$ \\ ${ }^{1}$ Physics Department, Saratov State University, Astrakhanskaya Street 83, Saratov, 410026, Russia \\ ${ }^{2}$ Department of Physics, The Technical University of Denmark, 2800 Kongens Lyngby, Denmark \\ ${ }^{3}$ Department of Medical Physiology, Panum Institute, University of Copenhagen, 2200 Copenhagen N, Denmark
}

(Received 29 July 2002; published 20 December 2002)

\begin{abstract}
The individual functional unit of the kidney (the nephron) displays oscillations in its pressure and flow regulation at two different time scales: fast oscillations associated with a myogenic dynamics of the afferent arteriole, and slower oscillations arising from a delay in the tubuloglomerular feedback. We investigate the intra- and internephron entrainment of the two time scales. In addition to full synchronization, both wavelet analyses of experimental data and numerical simulations reveal a partial entrainment in which neighboring nephrons attain a state of chaotic synchronization with respect to their slow dynamics, but the fast dynamics remain desynchronized.
\end{abstract}

DOI: 10.1103/PhysRevE.66.061909

PACS number(s): 87.19.-j, 87.90.+y, 05.45.Xt, 05.45.Tp

\section{INTRODUCTION}

The concept of homeostasis [1], i.e., the ability of the body to maintain a nearly constant internal milieu despite changes in the external conditions, plays an essential role in description of physiological control systems.

It is sometimes assumed that homeostasis implies that the physiological variables are kept near a stable steady state by means of effective feedback regulation. While this may be the case in certain situations, biological systems in general should be considered as open dissipative systems that are maintained under far-from-equilibrium conditions [2]. Regular and irregular oscillations associated with various forms of instability are common features of behavior that can be observed during normal functioning or arise in connection with particular states of disease [3].

Hormonal control systems, for instance, often show pulsating dynamics with insulin, testosterone, growth hormone, and several other hormones being released in pulses at $1-3 \mathrm{~h}$ intervals [4]. The release of insulin also displays a more rapid oscillatory pattern with periods in the $8-15$ min range. These oscillations, which are most readily observed in the portal vein between the pancreas and the liver, may be associated with oscillations of the intracellular $\mathrm{Ca}^{++}$concentration in the pancreatic $\beta$ cells [5]. The $\beta$ cells also exhibit a much faster form of bursting dynamics, with a rapid spiking behavior of their membrane potentials interrupted by silent periods [6]. Similar dynamics, related to changes in the ionic currents that cross the cell membrane, are found in the response of certain sensory nerve cells [7].

The kidneys play an important role in regulating the blood pressure and maintaining a proper environment for the cells of the body. At the same time, to protect its function against variations in the arterial blood pressure, the individual functional unit of the kidney (the nephron) has a feedback mechanism (the so-called tubuloglomerular feedback) that regulates the incoming blood flow depending on the $\mathrm{NaCl}$ concentration of the fluid that leaves the nephron. Experiments by Leyssac and Holstein-Rathlou [8,9] have demonstrated that this feedback regulation can become unstable and generate self-sustained oscillations in the proximal intratubu- lar pressure with a typical period of 30-40 s. With different amplitudes and phases the same oscillations are manifest in the distal intratubular pressure and in the chloride concentration near the terminal part of the loop of Henle [10]. While for normal rats the oscillations have the appearance of a limit cycle with a sharply peaked power spectrum [Fig. 1(a)], highly irregular oscillations, displaying a broadband spectral distribution with significant subharmonic components, are observed for spontaneously hypertensive rats [Fig. 1(b)] [8]. Different forms of entrainment between the tubular pressure variations in adjacent nephrons were described in two recent publications $[11,12]$. Observation of both in-phase and antiphase synchronization was reported for the regular pressure oscillations in normal rats while spontaneously hypertensive rats revealed signs of chaotic phase synchronization.
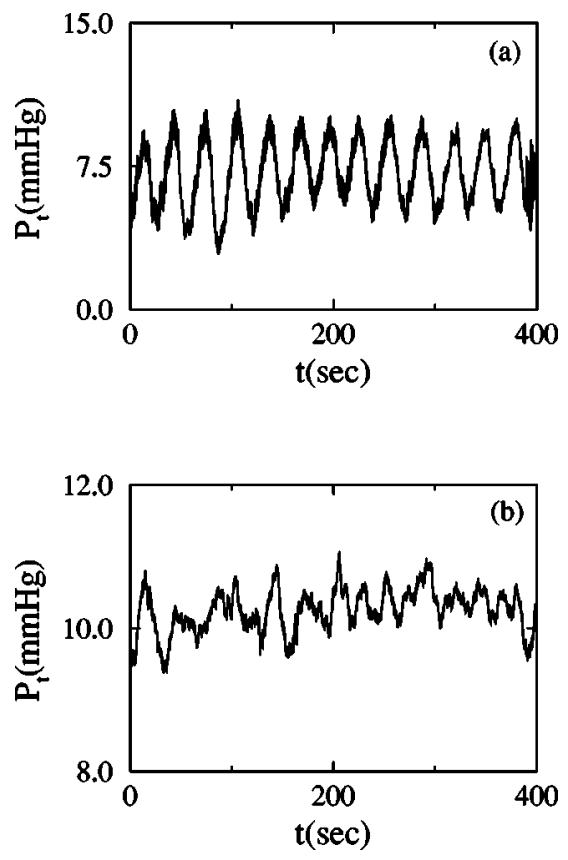

FIG. 1. Regular tubular pressure oscillations from a normotensive rat (a) and irregular pressure variations from a spontaneously hypertensive rat (b). 
Entrainment phenomena of this type are of considerable interest from a physiological point of view. It is known, for instance, that epileptic seizures are related to the synchronization of larger groups of cells in the brain [13]. For the kidney, the aggregate response of the ensemble of nephrons is expected to depend on their state of synchronization. If the nephrons operate in unison, a clear and immediate response is expected to changes, for instance, in the arterial pressure. Without synchronization, on the other hand, a slower and less pronounced response is likely to occur.

While entrainment of single-mode deterministic or stochastic oscillations is well understood, the dynamics of systems with several oscillatory modes is less studied. Living systems often exhibit oscillations with different time scales. The thalamocortical relay neurons, for instance, can generate either spindle or delta oscillations [14] and Neiman and Russell [15] have recently found that the electroreceptors in paddlefish can be biperiodic. In the present paper we describe the individual nephron as a two-mode oscillator demonstrating relatively fast oscillations associated with the myogenic regulation of the arteriolar diameter and slower oscillations related to the delay in tubuloglomerular feedback. We study numerically as well as experimentally the entrainment between these time scales both within the individual nephrons and between neighboring nephrons.

\section{NEPHRON AUTOREGULATION}

\section{A. Wavelet analysis}

Signals generated by living systems are typically nonstationary and inhomogeneous, and processing of such signals by means of conventional techniques such as Fourier analysis can lead to problems with respect to the interpretation of the results obtained. Among the various approaches developed to study nonstationary data, wavelet analysis is probably the most popular [16]. In particular, this method gives us the possibility of investigating the temporal evolution of signals with different rhythmic components.

The wavelet transform of a signal $x(t)$ is performed as follows:

$$
T_{\psi}[x](a, b)=\frac{1}{\sqrt{a}} \int_{-\infty}^{\infty} x(t) \psi^{*}\left(\frac{t-b}{a}\right) d t
$$

where $\psi$ is a "mother" function that in general can have an arbitrary shape provided it is solitonlike with zero average. $T_{\psi}[x](a, b)$ are the wavelet coefficients, $a$ being a time scaling and $b$ a time displacement parameter. In the analysis of various rhythmic contributions, the so-called Morlet wavelet is often applied:

$$
\psi(\tau)=\exp \left(j k_{0} \tau\right) \exp \left[-\frac{\tau^{2}}{2}\right]
$$

In the present study we shall use this function with $k_{0}=2 \pi$ and $\tau=(t-b) / a$.

In addition to the wavelet transform coefficients $T_{\psi}[x](a, b)$ we can estimate the energy density $E_{\psi}[x]$ $\times(a, b)=\left|T_{\psi}[x](a, b)\right|^{2}$. The result there is a three-
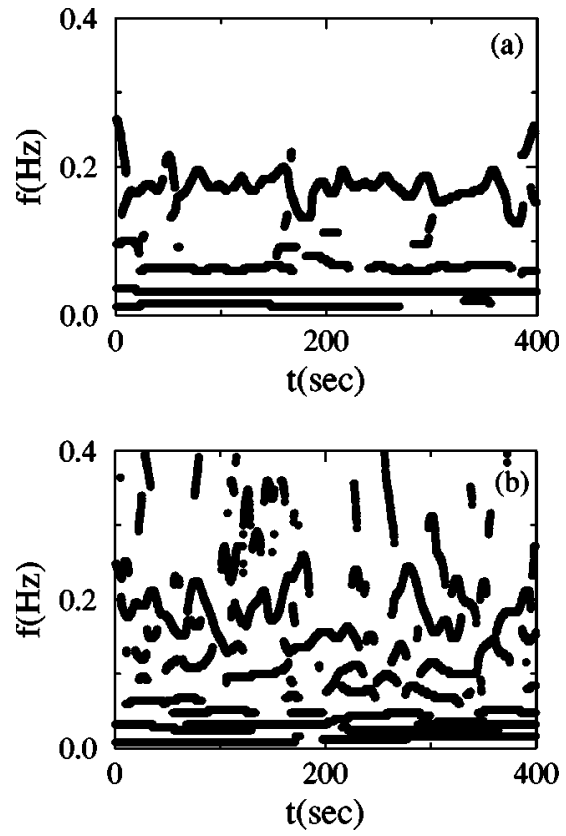

FIG. 2. Wavelet analysis of the two time series presented in Fig. 1.

dimensional surface $E_{\psi}[x](a, b)$ or $E_{\psi}[x](f, b)$, where $f$ is the frequency $(f=1 / a)$. Sections of this surface at fixed time moments $b=t_{0}$ correspond to the local energy spectrum. To simplify the visualization of the time-scale spectrum $E_{\psi}[x](f, b)$ we can consider the dynamics of only the local maxima of $E_{\psi}[x]\left(f, t_{0}\right)$, i.e., the peaks of the local spectra. Figure 2 shows the different components detected in the time series of Fig. 1. Inspection of the figure reveals that the slow oscillations, whether they are periodic or chaotic, maintain a nearly constant frequency through the observation time. The fast oscillations, on the other hand, fluctuate around some average value. This may be related to a complex modulation of the fast oscillations by the slow dynamics or to the influence of noise (since the fast oscillations are small in amplitude, they are more sensitive to fluctuations). However, this picture does not give information about the dominant spectral components. This information can be obtained, for example, from a scalogram, i.e., a time averaged power spectrum, which is an analog to the Fourier power spectrum. Such a scalogram is illustrated in Fig. 3 where a wellpronounced peak around $0.03 \mathrm{~Hz}$, corresponding to the slow mode mediated by tubuloglomerular feedback mode, is distinguishable. The other peak at $0.15-0.2 \mathrm{~Hz}$ derives from the fast myogenic dynamics. It is interesting to note how clearly these oscillations can be detected from the tubular pressure variations. Since both the above frequency components are of physiological interest we extract them from the original wavelet transformation for further analysis of their coherence properties. Figure 4 displays the relation between fast and slow oscillations in a single nephron. For the periodic oscillations observed for normotensive rats [Fig. 4(a)], the fast and slow components adjusted their periods in accordance with one another to maintain a 1:4 entrainment during the 

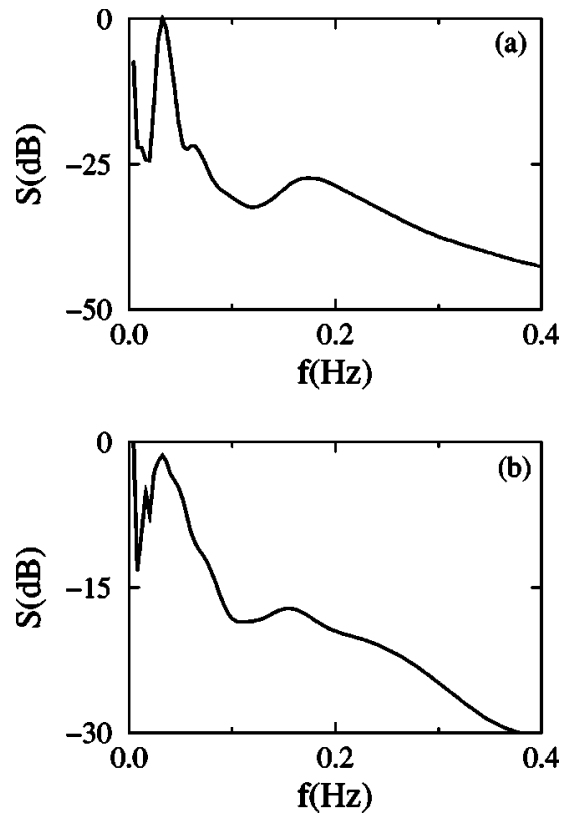

FIG. 3. Power spectrum obtained from the wavelet analysis for the two time series presented in Fig. 1. Two peaks, representing the fast myogenic oscillations and the slower tubuloglomerular oscillations, are well distinguished.

observation time. For the chaotic oscillations observed for hypertensive rats [Fig. 4(b)], the ratio changes more randomly in time.

\section{B. Model}

The functional unit of the kidney may be considered as a filtration device with an internal feedback control that regu-
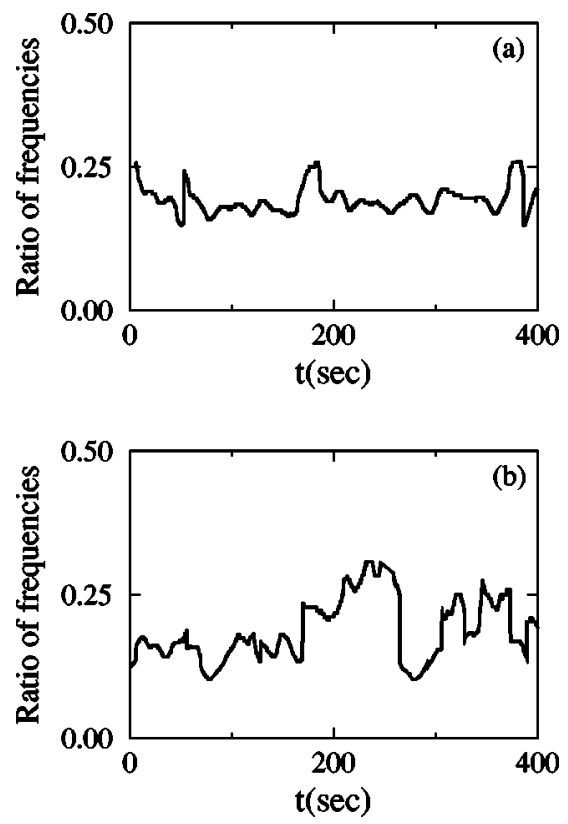

FIG. 4. Ratio of the internal time scales for a normotensive rat (a) and for a hypertensive rat (b). Note the 1:4 synchronization for the normotensive rat. lates the blood flow. The incoming blood flow passes through the afferent arteriole to the capillary system of the glomerulus where the filtration of water, salts, and small molecules into the proximal tubule takes place. As it leaves this tubule, the filtrate travels a significant distance through the loop of Henle where a variety of passive (osmotically driven) and active (enzymatically controlled) reabsorption and excretion processes take place. Before it leaves the tubular system, the fluid passes a group of specialized cells (the macula densa cells) that respond to variations in the $\mathrm{NaCl}$ concentration by eliciting a signal that influences the diameter of the afferent arteriole, providing the so-called tubuloglomerular feedback [17]. This is a negative feedback mechanism that serves to maintain the working conditions of the nephron relatively constant in spite of variations in the arterial blood pressure. Over the years significant efforts have been made to develop mathematical models that can account for the observed regular and irregular pressure variations and describe the physiological processes that occur along the tubular system [1822]. A particular aspect of this research has been to show that the transition from regular oscillations to irregular variations in the tubular pressure can be explained in terms of parameter changes within the framework of well-established physiological mechanisms. A review of the work may be found in the recent contribution by Andersen et al. [23]. Here, a model of nephron-nephron interaction was developed and it was shown that this model can produce a variety of different synchronization phenomena.

Autoregulation of the pressures and flows in the individual nephron may be described by the following model $[21,22]$ :

$$
\begin{aligned}
\dot{P}_{t} & =\frac{1}{C_{t u b}}\left\{F_{f}\left(P_{t}, r\right)-F_{r e a b}-\left(P_{t}-P_{d}\right) / R_{H}\right\}, \\
\dot{r} & =v_{r}, \\
\dot{v}_{r} & =\frac{1}{\omega}\left\{P_{a v}\left(P_{t}, r\right)-P_{e q}\left(r, \Psi\left(X_{3}, \alpha\right), T\right)-\omega d v_{r}\right\}, \\
\dot{X}_{1} & =\frac{1}{R_{H}}\left(P_{t}-P_{d}\right)-\frac{3}{T} X_{1}, \\
\dot{X}_{2} & =\frac{3}{T}\left(X_{1}-X_{2}\right), \\
\dot{X}_{3} & =\frac{3}{T}\left(X_{2}-X_{3}\right) .
\end{aligned}
$$

The first equation represents the pressure variations in the proximal tubule in terms of the in- and outgoing fluid flows. Here, $F_{f}$ is the single-nephron glomerular filtration rate and $C_{t u b}$ is the elastic compliance of the tubule. The flow into the loop of Henle is determined by the difference $\left(P_{t}-P_{d}\right)$ between the proximal and the distal tubular pressures and by the flow resistance $R_{H}$. The reabsorption in the proximal tubule $F_{\text {reab }}$ is assumed to be constant. 
The following two equations describe the dynamics associated with the flow control in the afferent arteriole. Here, $r$ represents the radius of the active part of the vessel and $v_{r}$ is its rate of increase. $d$ is a characteristic time constant describing the damping of the oscillations, $\omega$ is a measure of the mass relative to the elastic compliance of the arteriolar wall, and $P_{a v}$ denotes the average pressure in the active part of the arteriole. $P_{e q}$ is the value of this pressure for which the arteriole is in equilibrium with its present radius and muscular activation $\Psi$. The expressions for $F_{f}, P_{a v}$, and $P_{e q}$ involve a number of algebraic equations that must be solved along with the integration of Eq. (3).

The remaining equations in the single-nephron model describe the delay $T$ in the TGF regulation. This delay arises both from the transit time through the loop of Henle and from the cascaded enzymatic processes between the macula densa cells and the smooth muscle cells that control the contractions of the afferent arteriole. The feedback delay, which typically assumes a value of $12-18 \mathrm{~s}$, will be considered a bifurcation parameter in our analysis. Another important parameter is the strength $\alpha$ of the feedback regulation. This parameter takes a value of about 12 for normotensive rats, increasing to about 18 for hypertensive rats [24]. For a more detailed explanation of the model and the parameters (see Ref. [23]).

Both experimental investigations and our simulations [22] reveal one of the most important features of the singlenephron model, namely, the presence of two different time scales in the pressure and flow variations. Considering the model equations (3) we can identify the two time scales in terms of (i) a low-frequency (TGF-mediated) oscillation with a period $T_{h} \cong 2.2 T$ arising from the delay in the tubuloglomerular feedback, and (ii) somewhat faster oscillations with a period $T_{v} \approx T_{h} / 5$ associated with the inherent myogenic adjustment.

To determine $T_{h}$ and $T_{v}$ in our numerical simulations we have used the mean return times of the trajectory to appropriately chosen Poincaré sections

$$
T_{v}=\left\langle\left. T_{r e t}\right|_{v_{r}=0}\right\rangle \quad \text { and } \quad T_{h}=\left\langle T_{r e t} \mid \dot{X}_{2}=0\right\rangle .
$$

From these return times it is easy to calculate the intranephron rotation number (i.e., the rotation number associated with the two-mode behavior of the individual nephron)

$$
r_{v h}=T_{v} / T_{h}
$$

This measure will be used to characterize the various forms of frequency locking between the two modes. With varying feedback delay $T$ and varying slope $\alpha$ of the open loop feedback curve, Fig. 5 shows how the two oscillatory modes can adjust their dynamics and attain states with different rational relations $(n: m)$ between the periods. The regions of high resonances $(1: 4,1: 5$, and 1:6) are seen to exist in the physiologically interesting range of the delay time $T$ $\in[12 s, 20 s]$.

While the transitions between the different locking regimes always involve bifurcations, bifurcations may also occur within the individual regime. A period-doubling transi-

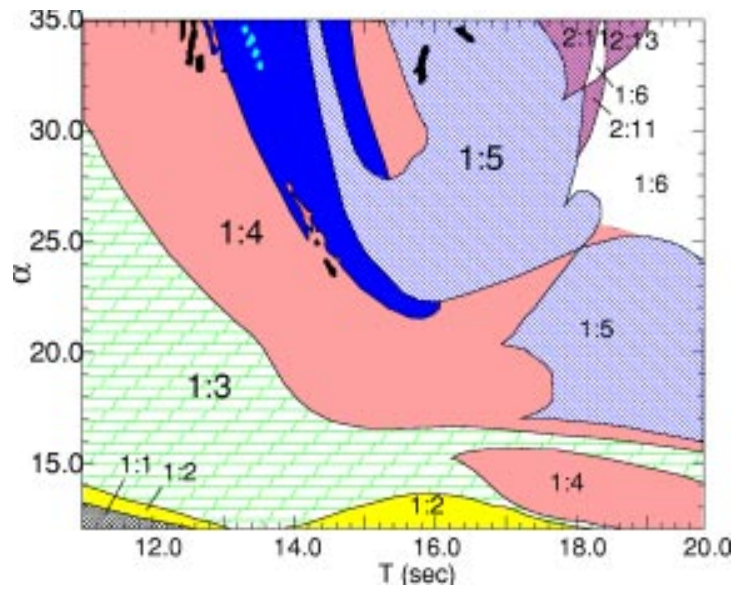

FIG. 5. Two-mode oscillatory behavior in the single-nephron model. Black colored regions correspond to a chaotic solution.

tion, for instance, does not necessarily change $r_{v h}$, and the intranephron rotation number may remain constant through a complete period-doubling cascade and into the chaotic regime. This is illustrated in Fig. 6 where we have plotted $r_{v h}$ as a function of the feedback gain $\alpha$ for different time delays $T_{2}=13.5 \mathrm{~s}$ (black circles) and $T=15.0 \mathrm{~s}$ (open circles). Phase projections $\left(P_{t}, r\right)$ from the various regimes are shown as insets. Inspection of the figure clearly shows that $r_{v h}$ remains constant under the transition from regular 1:4 oscillations (black circles for $\alpha=25.0$ ) to chaos (for $\alpha$ $=28.0$ ) (see insets 1 and 2). With further evolution of the chaotic attractor (inset 3), the 1:4 mode locking is destroyed. In the interval around $\alpha=31.5$ we observe 2:9 mode locking. A similar transition is observed for $T=15 \mathrm{~s}$ (open circles). Periodic 1:5 oscillations $(\alpha=27.0)$ evolve into a chaotic attractor ( $\alpha=28.5$ ), but the rotation number maintains a constant value. For fully developed chaos, the 1:5 locking again breaks down.

We conclude that, in addition to being regular or chaotic, the self-sustained pressure variations in the individual nephron can be classified as being synchronous or asynchronous with respect to the ratio between the two time scales that characterize the fast (arteriolar) mode and the slow (TGFmediated) mode, respectively. As we shall see, this complex-

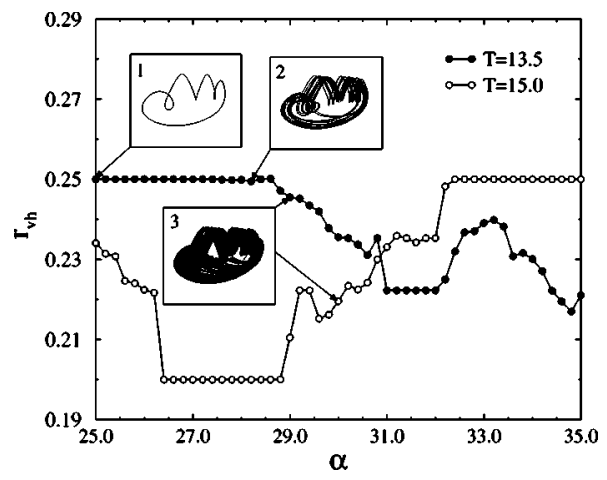

FIG. 6. Internal rotation number as a function of the parameter $\alpha$ calculated from the single-nephron model. Insets represent phase projections of typical regimes. 

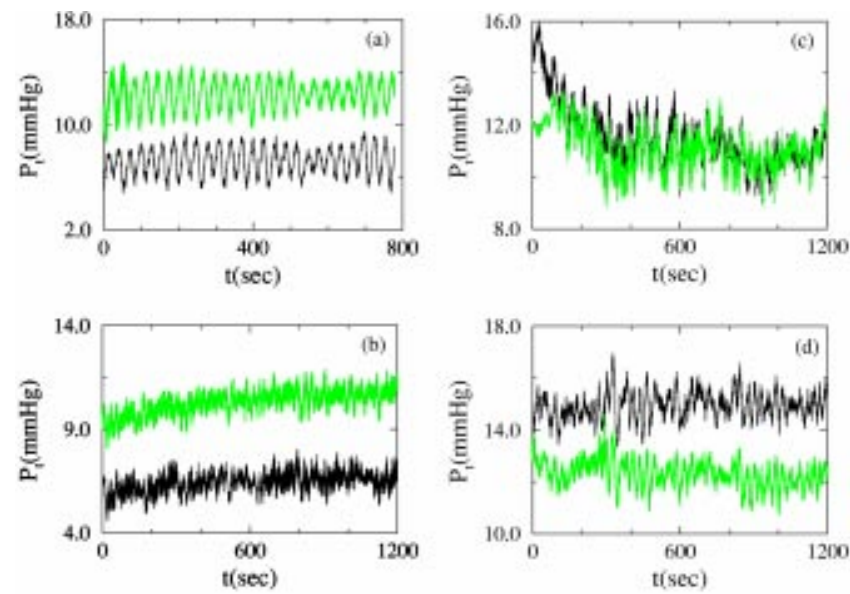

FIG. 7. Examples of the tubular pressure variation that one can observe in adjacent nephrons (a) for normotensive and (b)-(d) for hypertensive rats.

ity in behavior may play an essential role in the synchronization between a pair of interacting nephrons.

\section{ENTRAINMENT OF OSCILLATORY MODES FOR INTERACTING NEPHRONS}

\section{A. Experimental results}

Using anatomical criteria, neighboring nephrons having a high likelihood of deriving their afferent arterioles from the same interlobular artery were identified [25]. In these nephrons 29 out of 33 pairs (i.e., 80\%) were found to have synchronized oscillations. In contrast, nephron pairs not fulfilling these criteria only showed synchronous oscillations in one case out of 23 investigated pairs (i.e., 4\%). This observation shows that synchronized oscillations are preferentially found in nephrons originating from the same interlobular artery. Figure 7 displays the tubular pressure variations in pairs of neighboring nephrons for a normotensive rat (a) and for hypertensive rats (b)-(d). Oscillations presented in Figs. 7(b), 7(c), and 7(d) are significantly more irregular than the oscillations displayed in (a). One can visually observe a certain degree of synchronization between the interacting nephrons. It is difficult, though, to separately estimate the degree of adjustment for the myogenic oscillations and for the TGFmediated oscillations without special tools.

To study multimode interactive dynamics in coupled systems we propose to use the wavelet based coherence measure $\Gamma_{\Delta}$ (in analogy with the classical coherence function). Let $E_{\psi}[x x](f, t)$ and $E_{\psi}[y y](f, t)$ be the energy densities of signals $x(t)$ and $y(t)$. Let also in some range of frequencies $\Delta$ each of the processes $x(t)$ and $y(t)$ have a clearly expressed rhythm (e.g., the range of slow or fast oscillations for the two nephrons). In this case synchronization means that the corresponding frequencies for $x(t)$ and $y(t)$ will be locked (coincide). Such a situation corresponds to the value $\Gamma_{\Delta}=1$ for the function

$$
\Gamma_{\Delta}^{2}(t)=\frac{\max _{f \in \Delta}\left[E_{\psi}[x y](f, t)\right]^{2}}{\max _{f \in \Delta}\left[E_{\psi}[x x](f, t)\right] \max _{f \in \Delta}\left[E_{\psi}[y y](f, t)\right]} .
$$
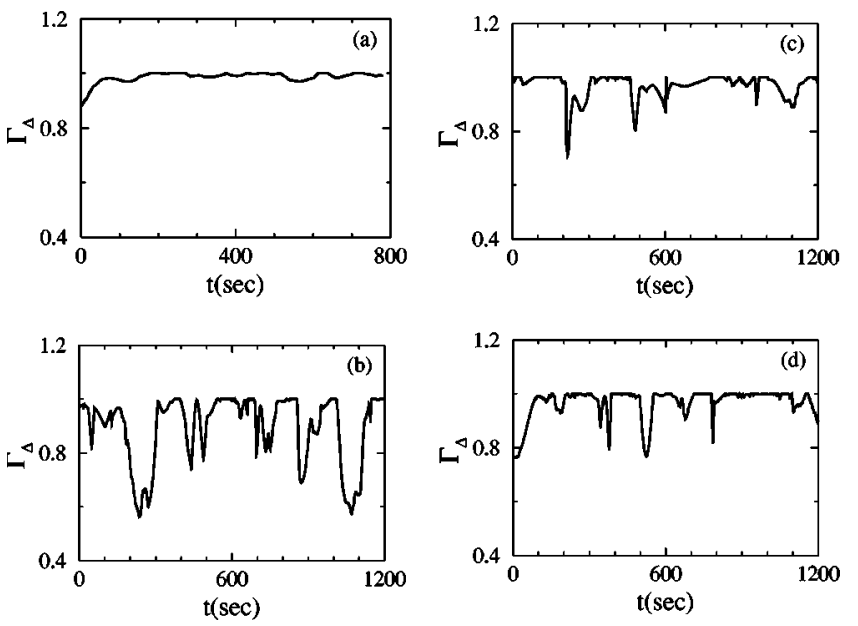

FIG. 8. Mutual wavelet analysis for the slow oscillations of the two time series presented in Fig. 7: (a) synchronous behavior; (b) nonsynchronous dynamics; (c) and (d) synchronous behavior but during limited time intervals.

Here, $E_{\psi}[x y](f, t)$ is the mutual energy density $E_{\psi}[x y](f, t)=\left|T_{\psi}[x y](f, t) T_{\psi}^{*}[y x](f, t)\right| . \Gamma_{\Delta}(t)$ is a function of time that allows us to follow the evolution of the interactive dynamics of the two processes in the chosen frequency range $\Delta$. The more synchronous the rhythms of these processes are, the closer $\Gamma_{\Delta}(t)$ will be to 1 .

Figures 8 and 9 demonstrate different degrees of coherence for the modes considered. For periodic oscillations (a), both the slow and fast modes of the interacting nephrons are perfectly locked during the observation time. For a system with complex oscillations subjected to noise one can speak about a certain degree of synchronization if the periods of locking are significant compared with the characteristic period of the oscillations [26]. Fully incoherent behavior with respect to both oscillatory modes can be observed in (b). In many cases we can diagnose synchronization of the slow motions (c),(d) for relatively long time intervals where the frequencies remain almost equal. The fast motions, on the
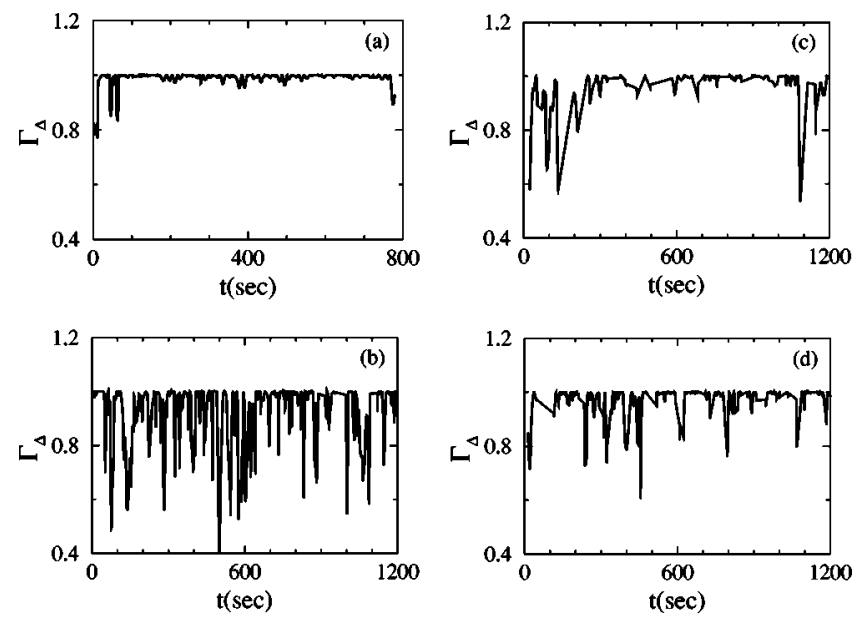

FIG. 9. Mutual wavelet analysis for the fast oscillations extracted from time series presented in Fig. 7. (a) and (c) illustrate synchronous behavior, (b) and (d) nonsynchronous dynamics. 
other hand, can demonstrate different coherence properties between nephrons. The oscillations can be locked during long periods of time together with the slow oscillations (c). We define this type of synchronization as full synchronization since all time scales of the system are locked. Another case (d) is when the fast oscillations are incoherent while the slow oscillations are synchronized during the time interval considered. We refer to this phenomenon as partial synchronization.

\section{B. Simulation results}

Neighboring nephrons can influence each other's blood supply either through vascularly propagated electrical (or electrochemical) signals or through a hemodynamic coupling arising via a direct redistribution of the blood flow between the coupled nephrons. While the hemodynamic coupling depends mainly on the flow resistances in the arteriolar network, the vascularly propagated coupling is associated with signal transmission between smooth muscle cells. The result is that only nephrons situated close to one another can interact via the vascularly propagated coupling. Nephrons situated farther apart but sharing a common piece of interlobular artery may interact via the hemodynamic coupling.

In the present work we shall focus our attention on the vascularly propagated coupling, assuming the hemodynamic coupling to be negligible. In the single-nephron model the equilibrium pressure in the afferent arteriole depends on the current radius $r$ and on the activation level $\Psi$ of the smooth muscles surrounding the arteriole and controlling its diameter. The muscular activation arises at the juxtaglomerular apparatus and travels upstream along the afferent arteriole in a damped fashion. When it reaches the branching point with the arteriole from the neighboring nephron, part of the signal may propagate down that arteriole and start to contribute to its TGF response. The coupling is considered nearly instantaneous since the time it takes for the vascular signal to reach the other nephron is very small relative to the period of the TGF oscillations. It has been observed [25] that the signal decreases nearly exponentially as it propagates. Thus only a fraction $\gamma=e^{-l / l_{0}}<1$ of the original activation level reaches the vascular smooth muscles close to macula densa of the neighboring nephron. In the expression for the vascular coupling parameter $\gamma, l$ is the propagation length of the coupling signal, and $l_{0} \cong 500 \mu \mathrm{m}$ is the characteristic length scale of the exponential decay. In the model, the vascularly propagated coupling is represented by adding a contribution of the activation level in one nephron to the activation level in the neighboring nephron:

$$
\Psi_{1,2}^{*}=\Psi_{1,2}+\gamma \Psi_{2,1}
$$

with $\gamma$ being the coupling parameter and $\Psi_{1,2}$ the uncoupled activation levels of the two nephrons as determined by their respective Henle flows. In view of the characteristic propagation length for the signal and of measured distances between neighboring nephrons along the arteriolar network, a typical value of $\gamma$ is considered to be $0.1-0.2$ [25]. By virtue

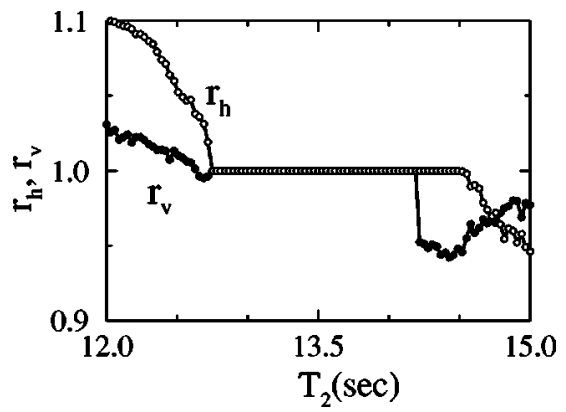

FIG. 10. Full and partial synchronization of fast and slow motions $\left(T_{1}=13.5 \mathrm{~s}, \alpha=30.0\right.$, and $\left.\gamma=0.06\right)$.

of the two-mode dynamics of the individual nephron, a number of interesting results appear.

The individual oscillatory system has two modes that can be locked with each other. However, an interaction between functional units can break their mutual adjustment. It is also plausible that a coupling can act in different ways on the fast and slow oscillations. For interacting systems we introduce two rotation numbers as follows:

$$
r_{v}=T_{v 1} / T_{v 2}, \quad r_{h}=T_{h 1} / T_{h 2} .
$$

To provide more information, the variation of the phase difference is calculated separately for the slow $h$ and for the fast $v$ oscillations.

Let us consider the case of $\alpha=30.0$ corresponding to a weakly developed chaotic attractor in the individual nephron. The coupling strength $\gamma$ and delay time $T_{2}$ in the second nephron are varied. Two different chaotic states can be recognized as asynchronous and synchronous (Fig. 10). For asynchronous behavior the rotation numbers $r_{h}$ and $r_{v}$ change continuously with $T_{2}$ while inside the synchronization region two cases can be distinguished. To the left, the rotation numbers $r_{h}$ and $r_{v}$ are both equal to unity since both slow and fast oscillations are synchronized. To the right $\left(T_{2}>14.2 \mathrm{~s}\right)$, while the slow $h$ mode of the chaotic oscillations remain locked, the fast $v$-mode drifts randomly. In this case the synchronization condition is satisfied only for one of oscillatory modes.

\section{CONCLUSIONS}

Based on the analysis of experimental results, we showed that the vascular dynamics and the tubuloglomerular feedback mechanism are responsible for two time scales associated with a fast and a slow oscillatory mode in the individual nephron. Both for periodic oscillations observed in normotensive rats and for the chaotic oscillations in hypertensive rats the two modes exhibit resonant behavior as well as nonsynchronous dynamics.

To investigate different types of internephron mode entrainment we developed a mutual wavelet transformation that allows us to easily analyze adjustments between different time scales from nonstationary data. We observed simultaneous (full) locking for the slow and fast oscillations both for 
normotensive and for hypertensive rats. We also identified a state of partial synchronization where the slow oscillations are synchronized while the fast motion demonstrates noncoherent behavior. Such a situation is typical for hypertensive rats.

Numerical simulations for coupled nephron models demonstrate similar behavior. With varying time delay in the tubuloglomerular feedback and varying strength of the vascu- lar coupling the experimentally observed forms of synchronous behavior were recovered.

\section{ACKNOWLEDGMENT}

This work was partly supported by INTAS Grant No. 012061 and RFBR Grant No. 01-02-16709. O.S. acknowledges support from the Natural Science Foundation of Denmark and INTAS Grant No. YS 2001/1-23.
[1] W. B. Cannon, Physiol. Rev. 9, 399 (1929).

[2] C. Nicolis and I. Prigogine, Self-Organization in Nonequilibrium Systems (Wiley, New York, 1977).

[3] L. Glass and M. C. Mackey, From Clocks to Chaos: The Rhythms of Life (Princeton University Press, Princeton, NJ, 1988).

[4] G. Leng, Pulsatility in Neuroendocrine Systems (CRC Press, Boca Raton, FL, 1988).

[5] A. Goldbeter, Biochemical Oscillations and Cellular Rhythms (Cambridge University Press, Cambridge, England, 1996).

[6] F. Ashcroft and P. Rorsman, Prog. Biophys. Mol. Biol. 54, 87 (1989).

[7] H. A. Braun, H. Wissing, K. Schäfer, and M. C. Hirsch, Nature (London) 367, 270 (1994).

[8] N.-H. Holstein-Rathlou and P. P. Leyssac, Acta Physiol. Scand. 126, 333 (1986).

[9] P. P. Leyssac and N.-H. Holstein-Rathlou, Pflügers Archiv. 407, 285 (1986).

[10] N.-H. Holstein-Rathlou and D. J. Marsh, Physiol. Rev. 74, 637 (1994).

[11] N.-H. Holstein-Rathlou, K.-P. Yip, O. V. Sosnovtseva, and E. Mosekilde, Chaos 11, 417 (2001).

[12] D. E. Postnov, O. V. Sosnovtseva, E. Mosekilde, and N.-H. Holstein-Rathlou, Int. J. Mod. Phys. B 15, 3079 (2001).

[13] F. Mormann, K. Lehnertz, P. David, and C. E. Elger, Physica D 144, 358 (2000).
[14] X.-J. Wang, Neuroscience 59, 21 (1994).

[15] A. Neiman and D. F. Russell, Phys. Rev. Lett. 86, 3443 (2001).

[16] A. Grossmann and J. Morlet, SIAM (Soc. Ind. Appl. Math.) J. Math. Anal. 15, 723 (1984); I. Daubechies, Ten Lectures on Wavelets (SIAM, Philadelphia, 1992).

[17] L. C. Moore, Am. J. Physiol. 247, F267 (1984).

[18] K. S. Jensen, E. Mosekilde, and N.-H. Holstein-Rathlou, Modens Develop. 54/55, 91 (1986).

[19] N.-H. Holstein-Rathlou and D. J. Marsh, Am. J. Physiol. 258, F1448 (1990).

[20] N.-H. Holstein-Rathlou and D. J. Marsh, Bull. Math. Biol. 56, 441 (1994).

[21] E. Mosekilde, Topics in Nonlinear Dynamics: Applications to Physics, Biology and Economic Systems (World Scientific, Singapore, 1996).

[22] M. Barfred, E. Mosekilde, and N.-H. Holstein-Rathlou, Chaos 6, 280 (1996).

[23] M. D. Andersen, N. Carlson, E. Mosekilde, and N.-H. Holstein-Rathlou, in Membrane Transport and Renal Physiology, edited by H. Layton and A. Weinstein (Springer, New York, 2001).

[24] P. P. Leyssac and N.-H. Holstein-Rathlou, Pflügers Arch. 413, 267 (1989).

[25] Y.-M. Chen, K.-P. Yip, D. J. Marsh, and N.-H. HolsteinRathlou, Am. J. Physiol. 269, F198 (1995).

[26] L. R. Stratonovich, Topics in the Theory of Random Noise (Gordon and Breach, New York, 1963). 